\title{
Effects of surface and interface scattering on anomalous Hall effect in Co/Pd multilayers
}

\author{
Z. B. Guo, ${ }^{1}$ W. B. Mi, ${ }^{2}$ R. O. Aboljadayel, ${ }^{1}$ B. Zhang, ${ }^{1}$ Q. Zhang, ${ }^{1}$ P. G. Barba, ${ }^{3}$ A. Manchon, ${ }^{3}$ and X. X. Zhang ${ }^{1, *}$ \\ ${ }^{1}$ Core Labs, King Abdullah University of Science and Technology (KAUST), Thuwal 23955-6900, Saudi Arabia \\ ${ }^{2}$ Tianjin Key Laboratory of Low Dimensional Materials Physics and Preparing Technology, Institute of Advanced Materials Physics, \\ Faculty of Science, Tianjin University, Tianjin 300072, China \\ ${ }^{3}$ Materials Science and Engineering, King Abdullah University of Science and Technology (KAUST), \\ Thuwal 23955-6900, Saudi Arabia
}

(Received 25 June 2012; revised manuscript received 30 August 2012; published 27 September 2012)

\begin{abstract}
In this paper, we report the results of surface and interface scattering on anomalous Hall effect in $\mathrm{Co} / \mathrm{Pd}$ multilayers with perpendicular magnetic anisotropy. The surface scattering effect has been extracted from the total anomalous Hall effect. By scaling surface scattering contribution with $\rho_{A H s} \sim \rho_{s s}^{\gamma}$, the exponent $\gamma$ has been found to decrease with the increase of surface scattering resistivity, which could account for the thickness-dependent anomalous Hall effect. Interface diffusion induced by rapid thermal annealing modifies not only the magnetization and longitudinal resistivity but also the anomalous Hall effect; a large exponent $\gamma \sim 5.7$ has been attributed to interface scattering-dominated anomalous Hall effect.
\end{abstract}

DOI: 10.1103/PhysRevB.86.104433

PACS number(s): 75.70.Cn, 73.63.-b

\section{INTRODUCTION}

Anomalous Hall effect (AHE) in magnetic materials has attracted intensive interest due to its fundamental physics related to spin-dependent transport and technical applications for field sensors. ${ }^{1-3}$ The Hall resistivity $\left(\rho_{H}\right)$ was found to follow the form of

$$
\rho_{H}=R_{o} B+4 R_{S} M,
$$

where the first term is the ordinary Hall resistivity due to the Lorentz force and the second term is anomalous Hall resistivity $\left(\rho_{A H}\right)$, which is proportional to the out-of-plane magnetization (M) of the film. ${ }^{3}$ The origin of AHE has been attributed to extrinsic and intrinsic mechanisms. The extrinsic mechanisms include skew scattering and side jump, both of which are due to the impurity and disorder scattering. ${ }^{4-6}$ In contrast, the intrinsic mechanism is related to the Berry phase of Bloch electrons, which is scattering independent, ${ }^{7,8}$ and has been found in some single crystals and epitaxial films, such as $\mathrm{CuCr}_{2} \mathrm{Se}_{4-x} \mathrm{Br}_{x}{ }^{9}$ and $\mathrm{SrRuO}_{3} \cdot{ }^{10,11}$ Since in realistic ferromagnetic systems, these mechanisms generally coexist, it is challenging to distinguish between them. ${ }^{12,13}$ The correlation between the anomalous Hall coefficient $R_{s}$ in Eq. (1) and zero-field longitudinal resistivity $\rho_{x x}$ is usually expressed by $R_{s} \propto \rho_{x x}^{\gamma}$, where the exponent $\gamma$ depends on the predominant mechanism. $\gamma=1$ has been observed in a few low-resistivity dilute ferromagnetic alloys at low temperatures, which indicates AHE is dominated by skew scattering. ${ }^{4,5,12}$ In most experiments, $\gamma \approx 2$ has been observed in relatively high-resistivity magnets due to the side-jump mechanism. ${ }^{6,14-16}$

Remarkably, in some heterogeneous ferromagnetic systems, $\gamma>2$ has been observed, for example, $\gamma=2.6$ in $\mathrm{Co} / \mathrm{Pt}$ and $\mathrm{Fe} / \mathrm{Cr}$ multilayers ${ }^{17,18}$ and $\gamma=3.7$ in granular Co-Ag films; ${ }^{19}$ the large exponent $\gamma$ has not been expected by the existing theories. All of these experiments have suggested the importance of spin-dependent interface scattering on AHE. The theoretical calculation on magnetic multilayers by Zhang has found that the scaling relation could be different from that of homogeneous magnetic materials. Considering the side jump, the power $\gamma$ in the scaling law $\rho_{A H} \propto \rho_{x x}^{\gamma}$ can be smaller, greater, or equal to two, which is dependent on both of the mean-free path and thickness of the ferromagnetic and nonmagnetic layers. ${ }^{20}$ In Zhang's analysis, interface scattering on AHE is neglected for simplicity; however, experimental results in the $\mathrm{Fe} / \mathrm{Cr}$ and $\mathrm{Co} / \mathrm{Pt}$ multilayers have demonstrated the dominated role of spin-dependent interface scattering on AHE. ${ }^{17,18}$

In this paper, we studied the effects of surface and interface scattering on $\mathrm{AHE}$ in a series of $[\mathrm{Co}(3 \AA) / \mathrm{Pd}(5 \AA)]_{n}$ multilayers with perpendicular magnetic anisotropy (PMA). The origins of the PMA in $\mathrm{Co} / \mathrm{Pd}$ multilayers have been attributed to the broken symmetry of Co atoms at the $\mathrm{Co} / \mathrm{Pd}$ interfaces ${ }^{21,22}$ and/or the magnetoelastic properties of Co-Pd interfacial alloys. ${ }^{23,24}$ Experimentally, it is challenging to distinguish the two mechanisms, because of the complexity at the interfaces. ${ }^{25,26}$ Rapid thermal annealing, which induces interface diffusion, should not only significantly modify the $\mathrm{M}$, anisotropy, and longitudinal resistivity but also AHE, if AHE is dominated by interface scattering in the multilayers. Indeed, such a correlation among M, longitudinal resistivity, and AHE has been observed in our experiment. The asdeposited multilayers present large values of $\mathrm{M}$ and $\rho_{x x}$, and high-temperature annealing induces interface diffusion, resulting in a decrease in $\gamma, \mathbf{M}$, and $\rho_{x x}$.

\section{EXPERIMENTS}

A series of $\mathrm{Co} / \mathrm{Pd}$ multilayers with the structure of $\operatorname{Pd}(50 \AA) /[\operatorname{Co}(3 \quad \AA) / \operatorname{Pd}(5 \AA)]_{n}$ were deposited by using magnetron sputtering on oxidized $\mathrm{Si}(100)$ substrates, with the $\mathrm{Co} / \mathrm{Pd}$ bilayers' repetition $n$ of $6,10,20,40,60$, and 80 . The deposition rates of $\mathrm{Co}$ and $\mathrm{Pd}$ are 0.40 and $0.59 \AA / \mathrm{sec}$, respectively. A 10 -nm-thick $\mathrm{SiO}_{2}$ layer was deposited on the top of the films to prevent oxidation of the $\mathrm{Co} / \mathrm{Pd}$ multilayers. Hall bars were fabricated by the shadow mask method. There are five contacts in the Hall bar geometry, which allow us to measure both the transverse and longitudinal resistance on the same sample simultaneously. Rapid thermal annealing was conducted in the temperatures ranging from 150 to $500^{\circ} \mathrm{C}$ in an argon atmosphere to induce interface diffusion. After 
$20 \mathrm{sec}$ to rise to the specified annealing temperature, the temperature remained for $2 \mathrm{~min}$. $\mathrm{M}$ and magnetotransport measurements were carried out using the Quantum Design S-VSM and physical properties measurement system (PPMS$9 \mathrm{~S})$, respectively, in the temperature range of 5-350 K and under magnetic fields up to $5 \mathrm{~T}$ applied perpendicular to the film plane. The layered periodic structures were examined with small angle $\mathrm{x}$-ray diffraction (XRD).

\section{RESULTS AND DISCUSSION}

\section{A. Magnetization and magnetotransport of the as-deposited multilayers}

The representative Hall loops of the as-deposited $[\mathrm{Co}(3 \AA) / \operatorname{Pd}(5 \AA)]_{n}$ multilayers, with repetition $n=6,20,40$, and 80 measured at $5 \mathrm{~K}$, are shown in Fig. 1(a). At high fields,
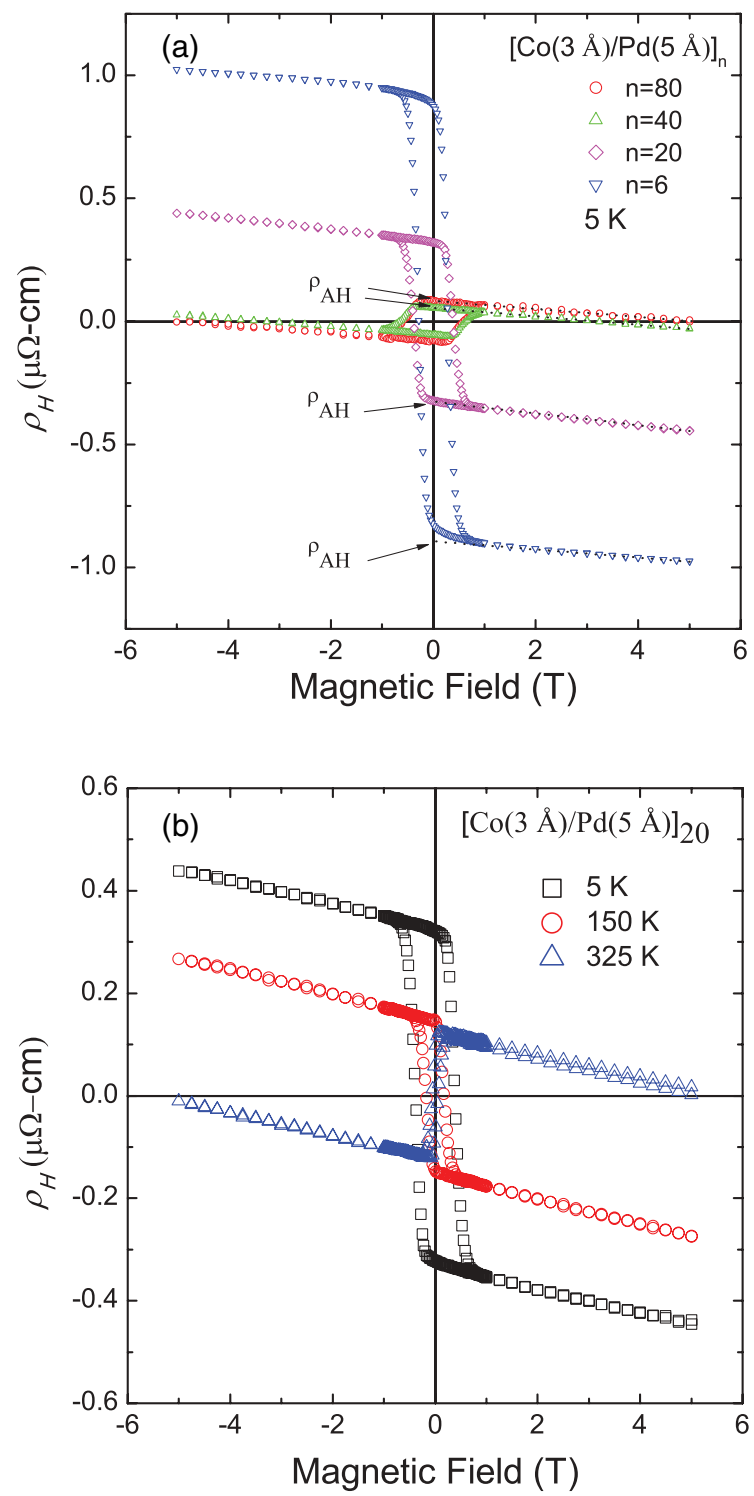

FIG. 1. (Color online) (a) The representative Hall loops of the as-deposited $[\operatorname{Co}(3 \AA) / \operatorname{Pd}(5 \AA)]_{n}$ multilayers with repetition $n=6$, 20, 40, and 80 measured at $5 \mathrm{~K}$. (b) The Hall loops of $[\mathrm{Co} / \mathrm{Pd}]_{20}$ multilayers measured at 5,150 , and $325 \mathrm{~K}$. the linear dependence of $\rho_{H}$ on field $H$ is attributed to the ordinary Hall effect, where the $\mathrm{M}$ is saturated. The slope of $\rho_{H}$ vs $H$ is negative, indicating an electronlike behavior, and the carrier density is in the order of $10^{22} / \mathrm{cm}^{3}$. All these multilayers present a square hysteresis loop, indicating the perpendicular anisotropy, with coercivity $\left(H_{c}\right)$ more than 2700 Oe. It is obvious that the squareness is reduced with decreasing $n$ due to the increase of the out-of-plane demagnetization field with decreasing the film thickness. The anomalous Hall resistivity $\rho_{A H}$ can be extracted from these loops by extrapolating the high-field data from the positive field to zero field, as shown in Fig. 1(a). The sign of AHE in $n=40$ and 80 is positive, while it is negative in $n=6$ and 20. The sign change with increasing repetition has also been observed by Rosenblatt et al. in $[\mathrm{Co}(2 \AA) / \operatorname{Pd}(9 \AA)]_{n}$ multilayers due to the opposite sign of AHE between interface and surface scattering. ${ }^{27}$

Shown in Fig. 1(b) are the Hall loops of $[\mathrm{Co} / \mathrm{Pd}]_{20}$ multilayers measured at 5, 150, and $325 \mathrm{~K}$; it exhibits an AHE sign change from negative to positive with the increase of temperature, which is attributed to the reduction in surface scattering because of the decrease in the mean-free path.

The temperature dependence of $\rho_{A H}$ and $\rho_{x x}$ for all the as-deposited multilayers is shown in Figs. 2(a) and 2(b), respectively. $\rho_{A H}$ increases with the rising temperature in these multilayers. The $\rho_{A H}-\mathrm{T}$ curve gradually shifts upward from negative to positive values with increasing repetition [Fig. 2(a)]. All of the films are metallic, and resistivity increases with decreasing the film thickness [Fig. 2(b)]. Figures 3(a) and 3(b) show the repetition dependence of zero-field longitudinal resistivity $\rho_{x x}$ and the saturated $\rho_{A H}$ at $5 \mathrm{~K}$, respectively. As $n$ decreases to less than $20, \rho_{x x}$ dramatically increases, which should be attributed to surface scattering. ${ }^{28,29}$ Where the change of the sign of AHE from positive to negative is accompanied with a sharp increase in the absolute magnitude $\left|\rho_{A H}\right|$. These phenomena indicate that (i) the sign of AHE for surface scattering is opposite to that of the bulk; it is positive for the bulk scattering, while negative for the surface scattering, and (ii) for the multilayers with $n \leqslant 20$, $\rho_{\mathrm{AH}}$ is dominated by surface scattering. As $n$ increases close to 80 , both $\rho_{x x}$ and $\rho_{A H}$ become $n$ independent, indicating the surface scattering could be ignored.

The dependence of $\rho_{A H}$ on $\rho_{x x}$ for the as-deposited multilayers at $5 \mathrm{~K}$ is shown in Fig. 4. One can find that $\rho_{A H}$ decreases with the increase of $\rho_{x x}$, accompanied by a sign change in $\rho_{A H}$, as has been discussed in Fig. 3. The inset in Fig. 4 shows the plot of $\log \left|\rho_{A H}\right|$ vs $\log \rho_{x x}$; these data do not follow a linear behavior. It is well known that a linear behavior usually presents in homogeneous ferromagnets without surface scattering. For the high-resistivity multilayers with $n \leqslant 20$, i.e., $\log \rho_{x x} \geqslant 2.38 \mu \Omega$-cm, surface scattering is dominant, and linear fitting yields $\gamma=1.24$. However, for the low-resistivity multilayers with $n \geqslant 40$, i.e., $\log \rho_{x x} \leqslant$ $2.07 \mu \Omega$-cm, $\rho_{A H}$ is positive, and $\log \left|\rho_{A H}\right|$ decreases with the increase of $\log \rho_{x x}$, leading to a negative $\gamma$, which is in contradiction to the theories. The origin should be attributed to the occurrence of the opposite surface scattering contribution on AHE as $n$ decreases. Therefore, for scaling analysis of the present $\mathrm{Co} / \mathrm{Pd}$ multilayers, it is necessary to separate the surface scattering and interface/bulk contributions, which will be carried out in the following sections. 

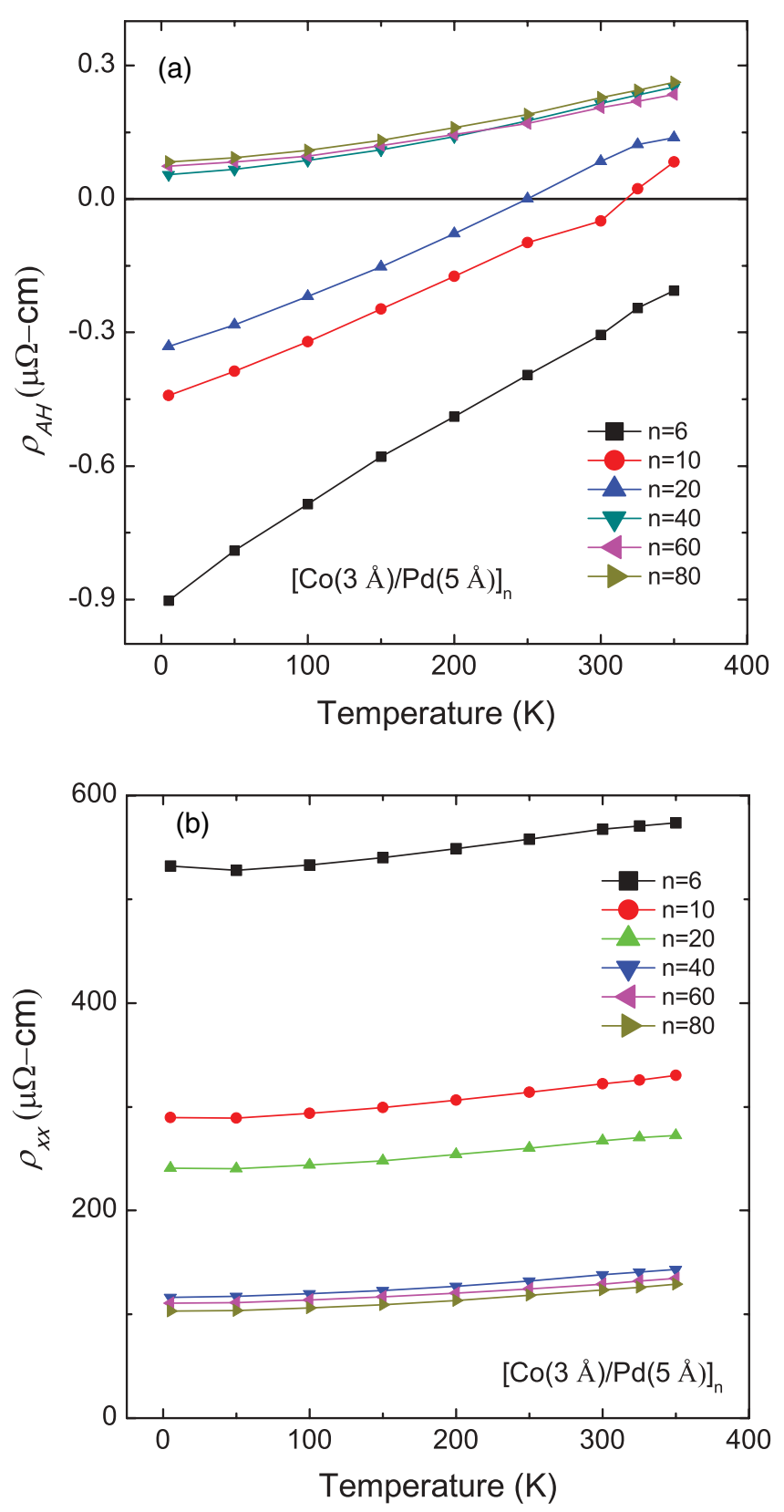

FIG. 2. (Color online) The temperature dependence of (a) $\rho_{A H}$ and (b) $\rho_{x x}$ for the as-deposited $[\operatorname{Co}(3 \AA) / \operatorname{Pd}(5 \AA)]_{n}$ multilayers, respectively.

\section{B. Scaling analysis of surface scattering effect}

Surface scattering, which induces obvious increases in resistivity, usually could be observed in thin films as the thickness is reduced comparable to the mean-free path. ${ }^{30}$ Experimentally, it has been found that the scattering at the surface, in the bulk, or at the interfaces contribute to AHE in different ways. The sign of surface scattering on AHE has been found to be same as that of the bulk in Ni films, ${ }^{28,29}$ while it is opposite to that of the bulk or interface scattering in CoAg granular films $^{31}$ and $\mathrm{Co} / \mathrm{Pd}$ multilayers. ${ }^{27}$

To identify the specific contributions to AHE, a simple scaling relation between $\rho_{A H}$ and $\rho_{x x}$ is given by

$$
\rho_{A H}=a \rho_{x x}+b \rho_{x x}^{2},
$$

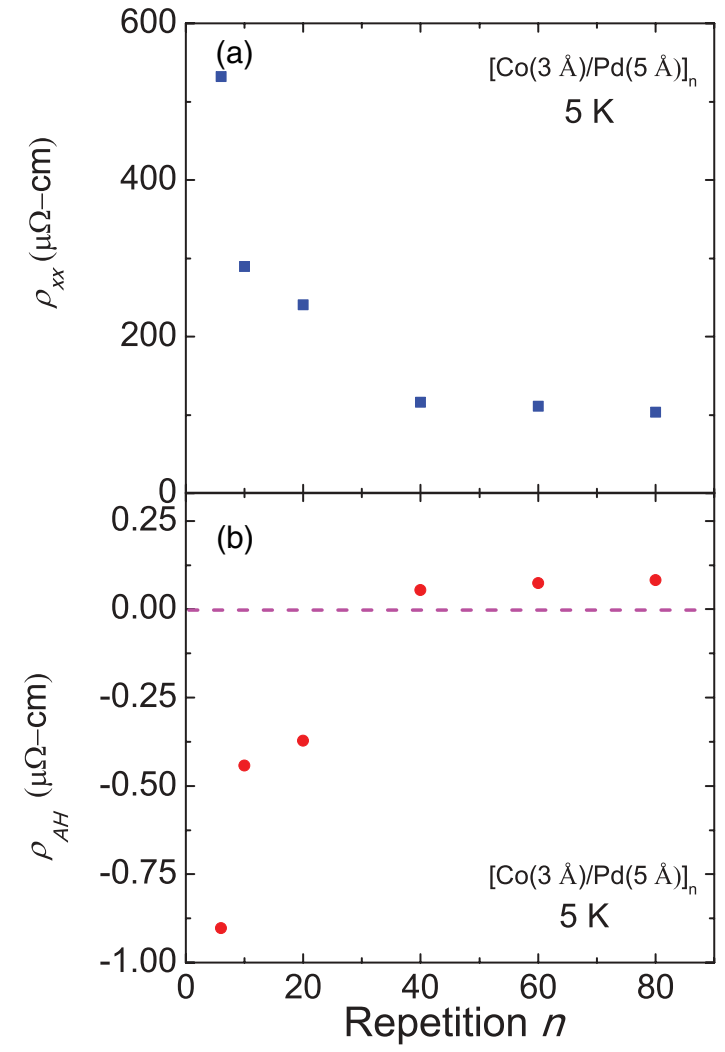

FIG. 3. (Color online) The repetition dependence of (a) $\rho_{x x}$ and (b) $\rho_{A H}$ at $5 \mathrm{~K}$ for the as-deposited $[\mathrm{Co}(3 \AA) / \operatorname{Pd}(5 \AA)]_{n}$ multilayers, respectively.

where the first term is related to the skew scattering and the second to the side jump. ${ }^{4-6}$ While plotting $\rho_{A H} / \rho_{x x}$ as a function of $\rho_{x x}$ for individual multilayers and linear fitting to the data, as an example shown in Fig. 5, one can find the coefficients $a$ and $b$, which are shown in the upper right insets in Fig. 5. The coefficient $a$ is negative, while the coefficient $b$ is positive, which is consistent with the observation in $\mathrm{Co} / \mathrm{Pt}$ multilayers, indicating that the skew scattering contribution to AHE is opposite to the side-jump contribution. ${ }^{17}$ As the film repetition decreases to less than 20 , where the surface scattering is present, a larger negative coefficient $a$ and smaller coefficient $b$ occur simultaneously. Based on Eq. (2), one could deduce the enhancement in skew scattering, while weakening in side jump due to the presence of surface scattering, but a theoretical explanation is still missing. A similar phenomenon is also observed in the Ni films. ${ }^{28,29,32}$ The lower right inset in Fig. 5 represents the repetition dependence for the data at $5 \mathrm{~K}$; the slope of low-resistivity films is significantly different from that of high-resistivity films, which present strong surface scattering; both of them are also different from the temperature dependence of the multilayers, as shown in the main frame of Fig. 5. Gerber et al. and Zhao et al. have raised questions for these distinguished characteristics, ${ }^{32,33}$ which could be understood in the present $\mathrm{Co} / \mathrm{Pd}$ multilayers considering a positive AHE from interface scattering and a negative AHE from surface scattering. For the temperature-dependent AHE, the contribution from interface could be increased with increasing temperature, because of the increase of longitudinal resistivity due to thermal activation 


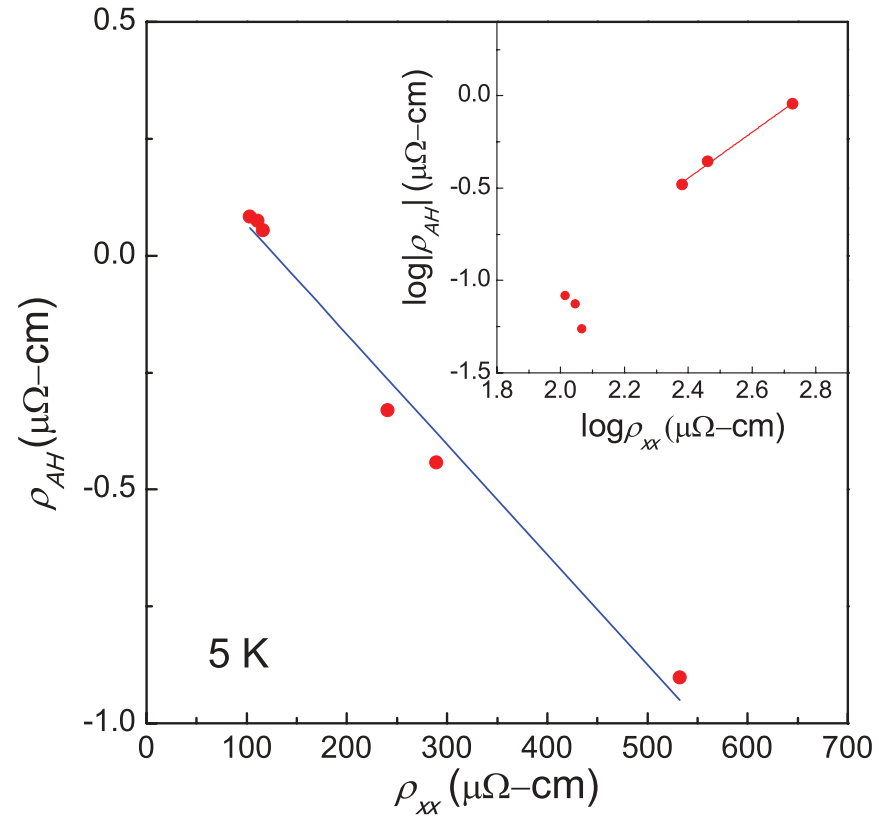

FIG. 4. (Color online) The dependence of $\rho_{A H}$ on $\rho_{x x}$ at $5 \mathrm{~K}$ for the as-deposited $[\mathrm{Co}(3 \AA) / \operatorname{Pd}(5 \AA)]_{n}$ multilayers. Inset: the plot of $\log \left|\rho_{A H}\right|$ vs $\log \rho_{x x}$.

induced phonon and magnon scattering. However, the opposite surface scattering contribution to AHE should be decreased due to the reduction in mean-free path, which results in positive slopes in Figs. 5 and 2(a). For the thickness-dependent AHE, as the thickness decreases, surface scattering contribution to AHE is significantly enhanced compared to that of the interface scattering, which could explain the negative slopes of the lines

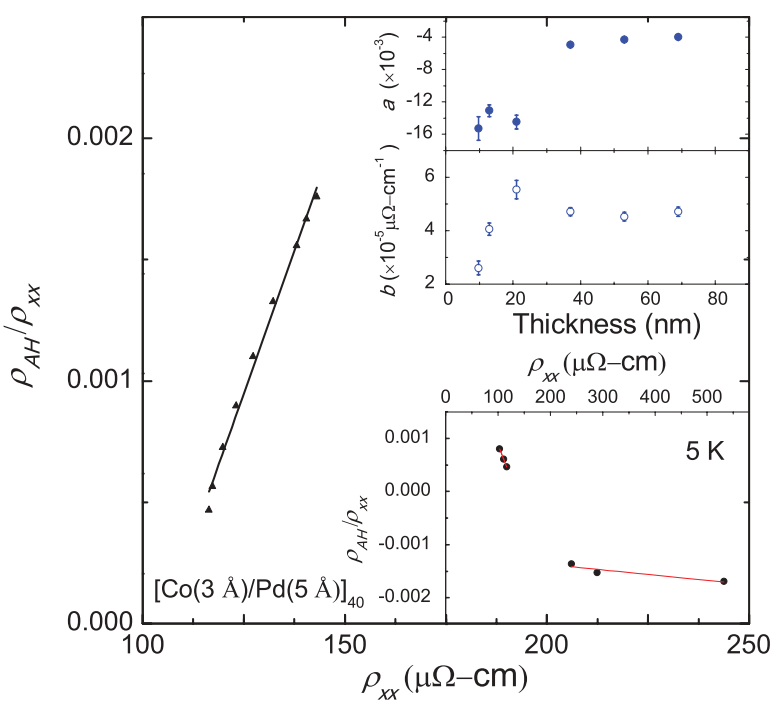

FIG. 5. (Color online) $\rho_{A H} / \rho_{x x}$ as a function of $\rho_{x x}$ for the asdeposited $[\mathrm{Co}(3 \AA) / \operatorname{Pd}(5 \AA)]_{40}$ multilayers. The black solid line is a linear fitting for temperature dependence of the sample. Upper right insets: dependence of coefficients $a$ and $b$ on the film thickness. Lower right inset: $\rho_{A H} / \rho_{x x}$ as a function of $\rho_{x x}$ at $5 \mathrm{~K}$ for the as-deposited $[\mathrm{Co}(3 \AA) / \operatorname{Pd}(5 \AA)]_{n}$ multilayers. The red solid lines are linear fittings for the data at $5 \mathrm{~K}$ for thickness dependence of the samples.

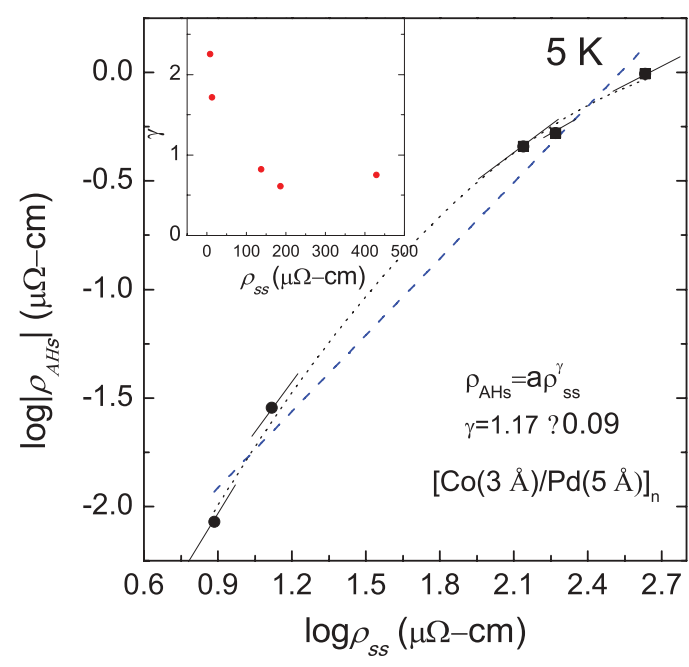

FIG. 6. (Color online) Analysis of the scaling relation by the form of $\left|\rho_{A H s}\right| \sim \rho_{s s}^{\gamma}$. The plot of $\log \left|\rho_{A H s}\right|$ vs $\log \rho_{s s}$. The dashed line is a linear fitting, yielding $\gamma=1.17 \pm 0.09$. The dotted line is a guide to the eye, and the short solid lines are the tangent line at each data point. Inset: the dependence of exponent $\gamma$ on $\rho_{s s}$.

in the lower right inset in Fig. 5, but it could not explain the less steep slope in high $\rho_{x x}$ than that in low $\rho_{x x}$, where the former presents much stronger surface scattering.

To extract the surface scattering contribution to AHE from the total values, Gerber et al. have proposed two formulas given by ${ }^{28}$

$$
\begin{gathered}
\rho_{s s}=\rho-\rho_{b}, \\
\rho_{A H s}=\rho_{A H}-\rho_{H b},
\end{gathered}
$$

where the surface scattering contribution to longitudinal resistivity $\rho_{s s}$ is described by the difference between the resistivity of a thinner film $\rho$ to that of a thicker film $\rho_{b}$. For the latter, the film is thick enough so that surface scattering could be ignored. The surface scattering contribution to AHE $\left(\rho_{A H s}\right)$ was done in a similar way, by subtracting the bulk contribution to AHE $\left(\rho_{A H b}\right)$ from the total AHE resistivity $\left(\rho_{A H}\right)$. Interestingly, the authors found that the scaling law for surface scattering follows $\rho_{A H S} \propto \rho_{s s}$. This scaling relation is the same as that of low-resistivity materials with the skew scattering mechanism, in which resistivity is less than $1 \mu \Omega$ $\mathrm{cm}$, while in the case of surface scattering, the $\rho_{s s}$ is more than hundreds of micro-ohm-centimeters.

For our series of samples, taking AHE resistivity and longitudinal resistivity of $[\mathrm{Co} / \mathrm{Pd}]_{80}$ as $\rho_{b}$ and $\rho_{A H b}$, respectively, we attempted to analyze the scaling relation by the form of $\rho_{A H s} \sim \rho_{s s}^{\gamma}$ for the $5 \mathrm{~K}$ data in Fig. 3. At $5 \mathrm{~K}$, phonon and magnon scattering could be ignored. Taking note that $\rho_{A H s}$ is negative, the plot of $\log \left|\rho_{A H s}\right|$ vs $\log \rho_{s s}$ is shown in Fig. 6; a linear fit, using all the data, gives $\gamma=1.17 \pm 0.09$. To a certain degree, it corresponds to $\gamma=1$ in $\mathrm{Ni}$ films and $\mathrm{Ni}-\mathrm{SiO}_{2}$ mixture. ${ }^{28}$ From the tangent line at each data point, one can find that the tendency of the slope decreases as $\rho_{s s}$ increases, indicating that $\gamma$ decreases with increasing $\rho_{s s}$. As shown in the inset of Fig. 6, $\gamma$ decreases from 2.2 to $\sim 0.7$ with an increase in $\rho_{s s}$. It is interesting that the behavior of $\gamma$ with respect to $\rho_{s s}$ resembles that of the thickness-dependent 


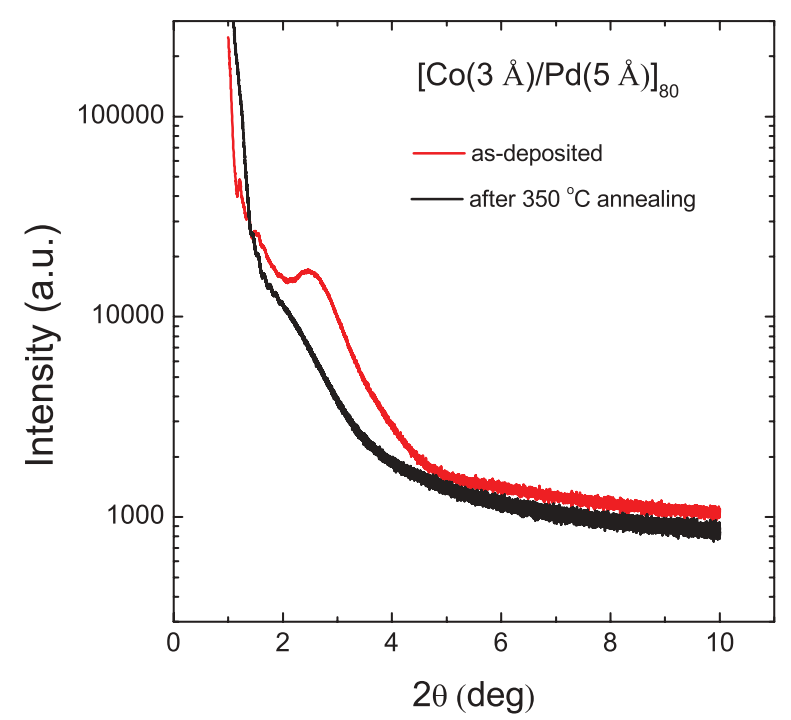

FIG. 7. (Color online) Small-angle XRD patterns for as-deposited and after annealing at $350^{\circ} \mathrm{C}$ for $2 \min$ for $[\operatorname{Co}(3 \AA) / \operatorname{Pd}(5 \AA)]_{80}$ multilayers. ( $\mathrm{Cu} \mathrm{K} \alpha$ radiation).

AHE shown in the lower right inset in Fig. 5; in a way, a smaller slope in $\rho_{A H} / \rho_{x x}$ vs $\rho_{x x}$ corresponds to a smaller $\gamma$. The decrease in $\gamma$ with increasing surface scattering should be attributed to the enhancement in surface-induced spin flip to randomize the spins, ${ }^{31,34}$ which could explain the phenomenon of the thickness-dependent AHE in Fig. 5.

\section{Interface scattering effect on AHE}

Now, we turn to focus on the AHE dependence on the interface diffusion in $[\mathrm{Co} / \mathrm{Pd}]_{80}$ multilayers induced by rapid thermal annealing. As discussed above, the surface scattering could be ignored in this sample. AHE should come from Co/Pd interface scattering and bulk scattering in the Co layer. In fact, the interface scattering is dominant in the multilayers, because rapid thermal annealing induced interface diffusion, resulting in $\rho_{x x}$ reduced by more than $70 \%$ after annealing at $500^{\circ} \mathrm{C}$ for 2 min. The advantage of using $\mathrm{Co} / \mathrm{Pd}$ multilayers other than $\mathrm{Cr} / \mathrm{Fe}$ and $\mathrm{Fe} / \mathrm{Cu}$ multilayers with in-plane easy-axis $\mathrm{M}$ for studying interface scattering on AHE is that the M and PMA in $\mathrm{Co} / \mathrm{Pd}$ multilayers are more sensitive to the interface. It is anticipated that any change in AHE related to the interface should also be reflected in the M and PMA.

To avoid the fluctuation from sample to sample, in this study, electrotransport and AHE measurements were carried on the same Hall sample, and $\mathrm{M}$ was measured on the same film, after successively annealing at the respective temperatures for $2 \mathrm{~min}$.

Small-angle XRD patterns of the as-deposited mulitilayers and the multilayers annealed at $350^{\circ} \mathrm{C}$ for $2 \mathrm{~min}$ are shown in Fig. 7. A clear fourth-order superstructure Bragg peak is exhibited in the as-deposited multilayers, and the peak is greatly suppressed after annealing at $350^{\circ} \mathrm{C}$ due to the interface diffusion.

Shown in Fig. 8 is the dependence of saturation magnetization $\left(M_{s}\right)$ and coercivity $\left(H_{c}\right)$ on the annealing temperature. The decrease in $M_{s}$ with increasing annealing temperature above $150^{\circ} \mathrm{C}$ should be attributed to the interface diffusion.

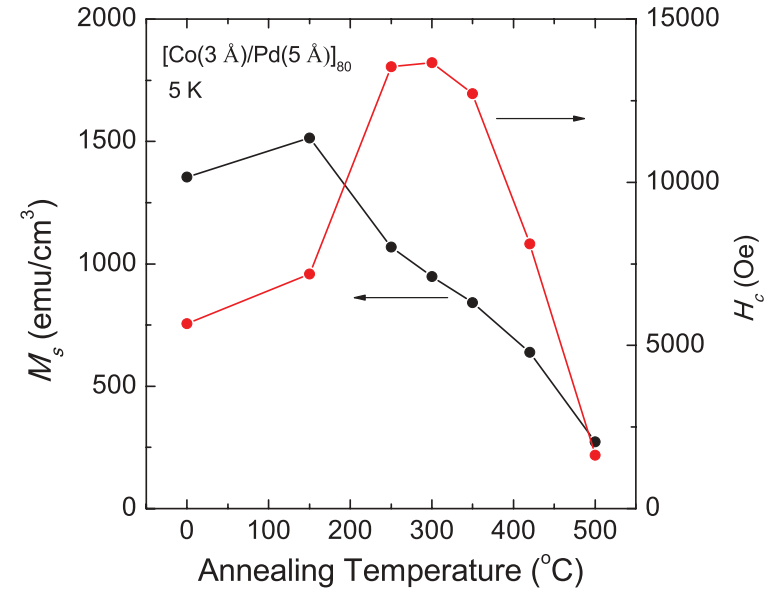

FIG. 8. (Color online) The dependence of saturation magnetization $\left(M_{s}\right)$ and coercivity $\left(H_{c}\right)$ on the annealing temperature for $[\mathrm{Co}(3 \AA) / \operatorname{Pd}(5 \AA)]_{80}$ multilayers.

However, the sharp increase in $H_{c}$ for annealing at lower temperatures below $300^{\circ} \mathrm{C}$ should be related to crystallization of the films.

The dependence of $\rho_{x x}$ and $\rho_{A H}$ measured at $5 \mathrm{~K}$ on annealing temperature is shown in Fig. 9. The decrease in $\rho_{x x}$ with the increase of annealing temperature above $150^{\circ} \mathrm{C}$ is correlated very well to the decrease in $M_{s}$ (Fig. 8), indicating that the interface diffusion reduces both the interface scattering and M. However, $\rho_{A H}$ exhibits a sharp increase.

To extract the influence of annealing on the scaling of AHE in $[\mathrm{Co} / \mathrm{Pd}]_{80}$ multilayers, not only should $\rho_{x x}$ and $\rho_{A H}$ be measured, but also the annealing-induced change in $M_{s}$ should also be taken into account. Shown in Figs. 10(a) -10(c) is the temperature dependence of $M_{s}, \rho_{x x}$ and $\rho_{A H}$ of the multilayers before and after annealing at various temperatures, respectively. The temperatures of these measurements are in the range from 5 to $350 \mathrm{~K}$. An increase in $M_{s}$ after annealing at $150{ }^{\circ} \mathrm{C}$ compared to that of the as-deposited sample [Fig. 10(a)], accompanied by a slight increase in $\rho_{x x}$ [Fig. 10(b)] should be attributed to the improvement of

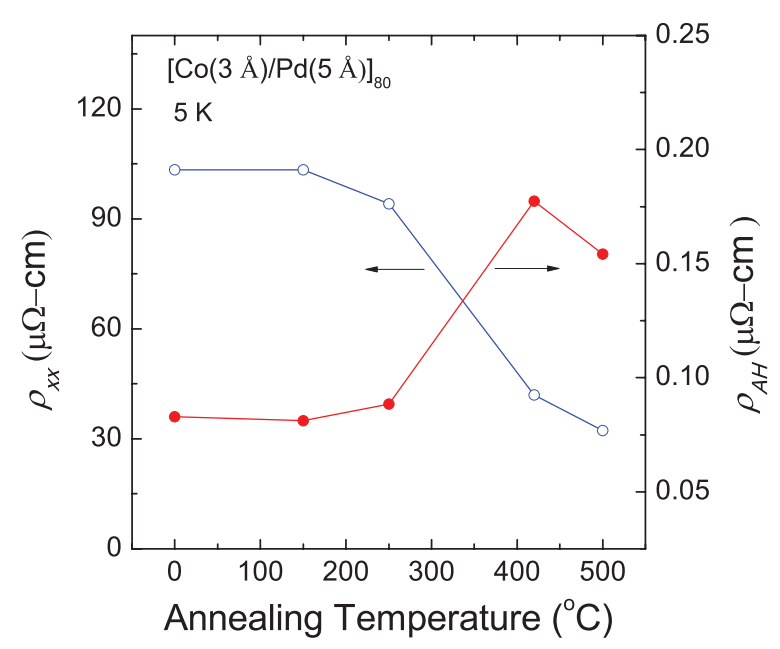

FIG. 9. (Color online) The dependence of $\rho_{x x}$ and $\rho_{A H}$ measured at $5 \mathrm{~K}$ on annealing temperature for $[\mathrm{Co}(3 \AA) / \operatorname{Pd}(5 \AA)]_{80}$ multilayers. 


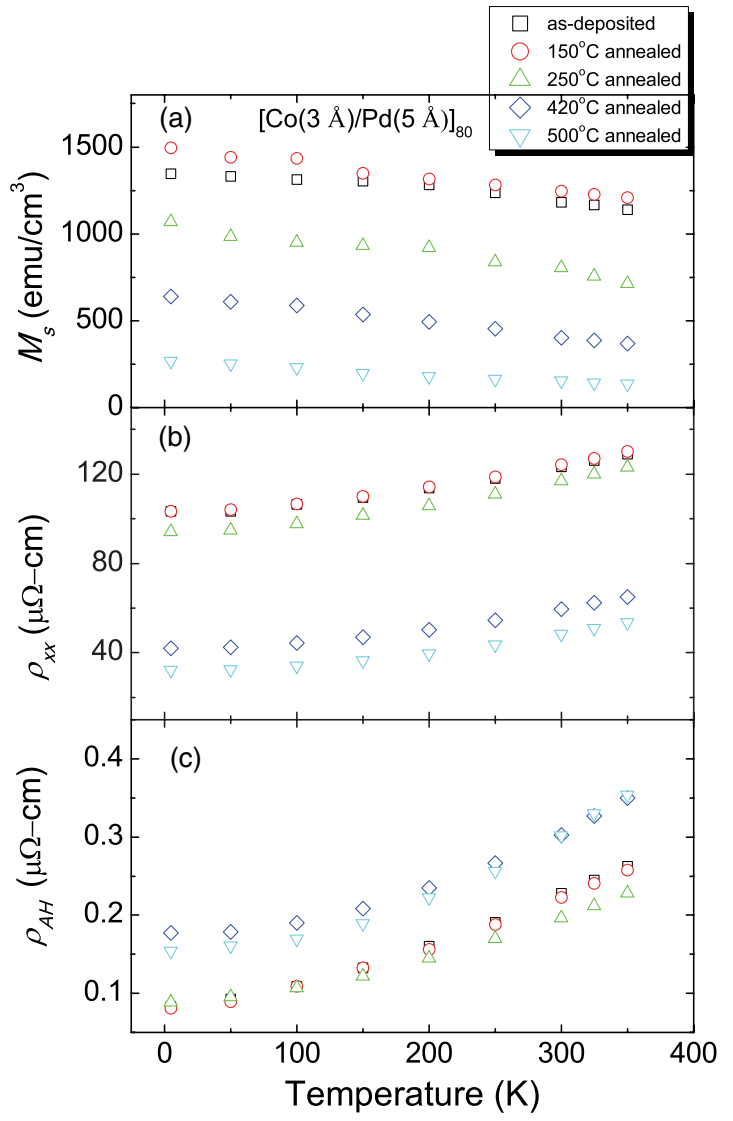

FIG. 10. (Color online) The temperature dependence of (a) $M_{s}$, (b) $\rho_{x x}$, and (c) $\rho_{A H}$ of the $[\operatorname{Co}(3 \AA) / \operatorname{Pd}(5 \AA)]_{80}$ multilayers before and after annealing at $150,250,420$, and $500{ }^{\circ} \mathrm{C}$.

the interfaces. Interface diffusion was obviously present and enhanced by further increases in the annealing temperature, resulting in the decreases of both $M_{s}$ and $\rho_{x x}$ for annealing above $250{ }^{\circ} \mathrm{C}$.

In Fig. 10(b), there is no significant difference in the tendency of the temperature-dependent $\rho_{x x}$ for respective annealing. However, different tendencies in the temperaturedependent $\rho_{A H}$ are observed in Fig. 10(c), exhibiting a cross in the curves for as-deposited and $250{ }^{\circ} \mathrm{C}$ annealing and a merge above $300 \mathrm{~K}$ for annealing at 420 and $500{ }^{\circ} \mathrm{C}$. It implies different $\gamma$ values for the similar tendencies in $\rho_{x x}$ vs T and different tendencies in $\rho_{A H}$ vs T. Shown in Fig. 11 is a typical plot of $\log R_{s} \mathrm{vs} \log \rho_{x x}$ for the multilayers after annealing at $420^{\circ} \mathrm{C}$, and the straight line in this figure is fitted by $R_{s} \sim \rho_{x x}^{\gamma}$; the slope of the fitting line represents the exponent $\gamma$. We plot the dependence of $\gamma$ with annealing temperature in the inset in Fig. 11; it exhibits a large $\gamma \sim 5.71 \pm 0.25$ in the as-deposited $[\mathrm{Co} / \mathrm{Pd}]_{80}$ multilayers and $\gamma \sim 5.76 \pm 0.34$ after annealing at $150{ }^{\circ} \mathrm{C}$. Subsequently, annealing at the higher temperatures of 420 and $500^{\circ} \mathrm{C}$ induces a reduction in the amplitude of $\gamma$ to $\sim 2.79 \pm 0.02$ and $2.86 \pm 0.10$, respectively. The decrease in $\gamma$ for annealing above $250{ }^{\circ} \mathrm{C}$ coincides with the decreases in $M_{s}$ (Fig. 8) and $\rho_{x x}$ (Fig. 9), which reflect the interface diffusion and alloying. In turn, the observation demonstrates that the large $\gamma \sim 5.7$ present in the as-deposited and $150^{\circ} \mathrm{C}$ annealed multilayers should be attributed to the interface characteristics of the magnetic multilayers. For comparison,

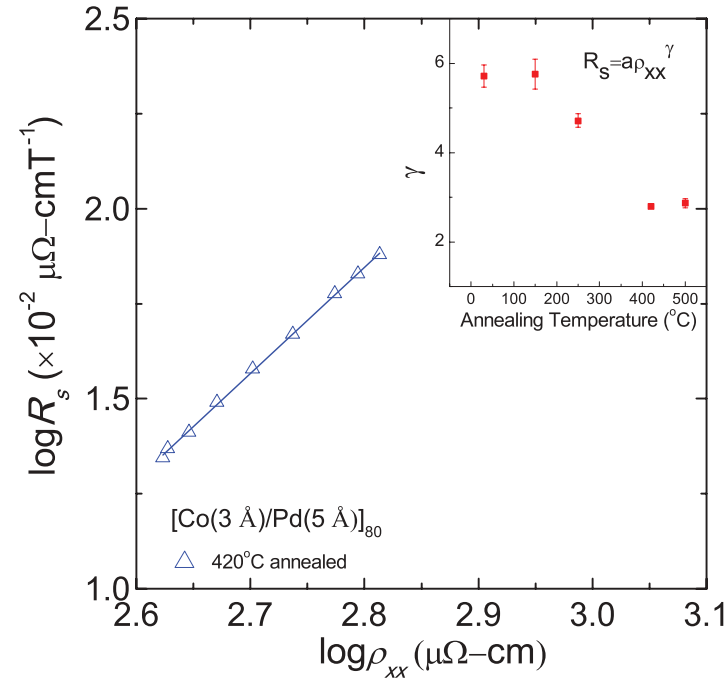

FIG. 11. (Color online) The plot of $\log R_{s}$ vs $\log \rho_{x x}$ for the $[\mathrm{Co}(3 \AA) / \operatorname{Pd}(5 \AA)]_{80}$ multilayers after annealing at $420^{\circ} \mathrm{C}$. The line is a linear fitting to the data. Inset: the dependence of the exponent $\gamma$ on annealing temperature for $[\mathrm{Co}(3 \AA) / \operatorname{Pd}(5 \AA)]_{80}$ multilayers.

in a homogenous system, the conventional skew scattering mechanism predicts $\gamma=1$, and both side-jump and intrinsic mechanisms yield $\gamma=2$. The large exponent $\gamma \sim 5.7$ in the current $\mathrm{Co} / \mathrm{Pd}$ multilayers, together with $\gamma \sim 2.6$ in $\mathrm{Co} / \mathrm{Pt}$ and $\mathrm{Fe} / \mathrm{Cr}$ multilayers ${ }^{17,18}$ and $\gamma \sim 3.7$ in Co-Ag granular films, ${ }^{19}$ indicates that interface scattering plays an important role in the AHE of these heterogeneous ferromagnetic systems.

\section{CONCLUSIONS}

Effects of surface and interface scattering on AHE have been studied in the $\mathrm{Co} / \mathrm{Pd}$ multilayers with perpendicular anisotropy. The samples present strong correlations not only between surface scattering and AHE but also among interface scattering, magnetism, and AHE. The effect of surface scattering on AHE has been studied in a series of as-deposited $\mathrm{Co} / \mathrm{Pd}$ multilayers with repetition from 6 up to 80 . The sign of surface scattering-induced AHE is opposite to that of interface scattering. The distinguished behaviors are present between temperature-dependent and thickness-dependent AHE. The former could be explained by thermal activation-induced electron-phonon and electron-magnon scattering, which enhances the positive AHE from bulk/interface scattering and leads to a positive slope. However, for the latter, reducing the multilayer repetition enhances the negative contribution of surface scattering to AHE, which results in a negative slope. By extracting the surface scattering resistivity and surface scattering-induced AHE resistivity from the total values, scaling analysis of the surface scattering components yields $\gamma \sim 1$ for the overall multilayer thickness. The amplitude of $\gamma$ for the individual multilayer was found to decrease by decreasing the multilayer repetition, which should be attributed to the enhancement in surface scattering-induced spin flip.

The interface scattering effect on AHE was studied based on $[\mathrm{Co} / \mathrm{Pd}]_{80}$ multilayers, where the surface scattering effect could be ignored, and a large exponent $\gamma \sim 5.7$ has been observed in the as-deposited and $150{ }^{\circ} \mathrm{C}$ annealed $\mathrm{Co} / \mathrm{Pd}$ 
multilayers. An increase of annealing temperature induces a decrease in $\gamma$, consistent with the decreases in $M_{s}$ and $\rho_{x x}$, demonstrating a large $\gamma$ is related to the interface scattering.

*Corresponding author: xixiang.zhang@kaust.edu.sa

${ }^{1}$ N. Nagaosa, J. Sinova, S. Onoda, A. H. MacDonald, and N. P. Ong, Rev. Mod. Phys. 82, 1539 (2010).

${ }^{2}$ L. Berger and G. Bergmann, The Hall Effect and Its Applications, edited by C. L. Chien and C. R. Westgate (Plenum Press, New York, 1980).

${ }^{3}$ Colin M. Hurd, The Hall Effect in Metals and Alloys (Plenum Press, New York, 1972).

${ }^{4}$ J. Smit, Physica (Amsterdam) 21, 877 (1955).

${ }^{5}$ J. Smit, Physica (Amsterdam) 24, 39 (1958).

${ }^{6}$ L. Berger, Phys. Rev. B 2, 4559 (1970).

${ }^{7}$ R. Karplus and J. M. Luttinger, Phys. Rev. 95, 1154 (1954).

${ }^{8}$ Z. Fang, N. Nagaosa, K. S. Tahakashi, A. Asamitsu, R. Mathieu,

T. Ogasawara, H. Yamada, M. Kawasaki, Y. Tokura, and K. Terakura, Science 302, 92 (2003).

${ }^{9}$ W. L. Lee, S. Watauchi, V. L. Miller, R. J. Vava, and N. P. Ong, Science 303, 1647 (2004).

${ }^{10}$ Y. Yao, L. Kleinman, A. H. MacDonald, J. Sinova, T. Jungwirth, D. S. Wang, E. Wang, and Q. Niu, Phys. Rev. Lett. 92, 037204 (2004).

${ }^{11}$ R. Mathieu, C. U. Jung, H. Yamada, A. Asamitsu, M. Kawasaki, and Y. Tokura, Phys. Rev. B 72, 064436 (2005).

${ }^{12}$ Y. Shiomi, Y. Onose, and Y. Tokura, Phys. Rev. B 79, 100404(R) (2009).

${ }^{13}$ Y. Tian, L. Ye, and X. Jin, Phys. Rev. Lett. 103, 087206 (2009).

${ }^{14}$ W. Jellinghaus and M. P. DeAndres, Ann. Phys. (Leipzig) 7, 189 (1961).

${ }^{15}$ J. P. Jan, Helv. Phys. Acta 25, 677 (1952).

${ }^{16}$ A. I. Schindler and E. Salkovitz, Phys. Rev. 99, 1251 (1955).

\section{ACKNOWLEDGMENTS}

We wish to thank J. Q. Li, Y. B. Yao, L. Chen, L. Li, and C. Zhao for sample fabrications and valuable discussions.

${ }^{17}$ C. L. Canedy, X. W. Li, and G. Xiao, Phys. Rev. B 62, 508 (2000).

${ }^{18}$ S. N. Song, C. Sellers, and J. B. Ketterson, Appl. Phys. Lett. 59, 479 (1991).

${ }^{19}$ P. Xiong, G. Xiao, J. Q. Wang, J. Q. Xiao, J. S. Jiang, and C. L. Chien, Phys. Rev. Lett. 69, 3220 (1992).

${ }^{20}$ S. Zhang, Phys. Rev. B 51, 3632 (1995).

${ }^{21}$ B. N. Engel, C. D. England, R. A. Van Leeuwen, M. H. Wiedmann, and C. M. Falco, Phys. Rev. Lett. 67, 1910 (1991).

${ }^{22}$ L. Néel, J. Phys. Radium 15, 376 (1954).

${ }^{23}$ R. Gontarz and T. Lucinski, J. Magn. Magn. Mater. 101, 253 (1991).

${ }^{24}$ S. K. Kim and S. C. Shin, J. Appl. Phys. 89, 3055 (2001).

${ }^{25}$ S. K. Kim and J. B. Kortright, Phys. Rev. Lett. 86, 1347 (2001).

${ }^{26}$ J. I. Hong, S. Sankar, A. E. Berkowitz, and W. F. Eglhoff, Jr., J. Magn. Magn. Mater. 285, 359 (2005).

${ }^{27}$ D. Rosenblatt, M. Karpovski, and A. Gerber, Appl. Phys. Lett. 96, 022512 (2010).

${ }^{28}$ A. Gerber, A. Milner, A. Finkler, M. Karpovski, L. Goldsmith, J. Tuaillon-Combes, O. Boisron, P. Mélinon, and A. Perez, Phys. Rev. B 69, 224403 (2004).

${ }^{29}$ Z. B. Guo, W. B. Mi, Q. Zhang, B. Zhang, R. O. Aboljadayel, and X. X. Zhang, Solid State Commun. 152, 220 (2012).

${ }^{30}$ E. H. Sondheimer, Adv. Phys. 1, 1 (1952).

${ }^{31}$ J. Q. Wang and G. Xiao, Phys. Rev. B 51, 5863 (1995).

${ }^{32}$ A. Gerber, A. Milner, L. Goldshmit, M. Karpovski, B. Lemke, H.-U. Habermeier, and A. Sulpice, Phys. Rev. B 65, 054426 (2002).

${ }^{33}$ J. Zhao, Y. J. Wang, X. F. Han, S. Zhang, and X. H. Ma, Phys. Rev. B 81, 172404 (2010).

${ }^{34}$ J. Chen and S. Hershfield, Phys. Rev. B 57, 1097 (1998). 